\title{
Gross motor skill acquisition in adolescents with Down syndrome
}

\author{
Sarah Meegan', Brian K.V. Maraj ${ }^{2}$, Daniel Weeks ${ }^{3}$ and Romeo Chua ${ }^{4}$ \\ 'Faculty of Health and Human Performance, Dublin City University, \\ ${ }^{2}$ Faculty of Physical Education and Recreation, University of Alberta, \\ ${ }^{3}$ Department of Psychology, Simon Fraser University, \\ ${ }^{4}$ School of Human Kinetics, University of British Columbia
}

\begin{abstract}
The purpose of this study was to assess whether verbal-motor performances deficits exhibited by individuals with Down syndrome limited their ability to acquire gross motor skills when given visual and verbal instruction together and then transferred to either a visual or verbal instructional mode to reproduce the movement. Nine individuals with Down syndrome $(6$ males, 3 females) performed 3 gross motor skills. Both visual and verbal instructional guidance was given to the participants over a 4-day period. Twenty-four hours later, the participants were video recorded as they produced the movements (used as baseline measures). On Day 6, they were randomly assigned into verbal and visual groups and required to reproduce the skills while the experimenter provided either visual demonstration or verbal instructions depending on the group. Based on skill performance scores, participants in the verbal-motor performance group demonstrated a lower level of proficiency and an increased number of performance errors when compared to participants in the visual-motor performance group. Moreover, while the visual group demonstrated an increase in performance levels compared to baseline measures, the opposite effect was seen for the verbal group.
\end{abstract}

Keywords: Motor learning, gross motor skills, visual demonstration, verbal instructions

Researchers have reported that individuals with Down syndrome initiate and complete movements more slowly and with greater variability than their peers without disabilities of a similar chronological age (e.g., Johnson and Olley, 1971). In addition to this, persons with Down syndrome exhibit general information processing difficulties as well as displaying a number of specific cognitive and motor problems when compared to other individuals with disabilities (Elliott, Gray and Weeks, 1991). Over the last 20 years, numerous studies have examined the effects of the unique Down syndrome karyotype on motor behaviour and development. More recently however, studies involving children and adults with Down syndrome have examined the influences of the Down syndrome karyotype on cerebral development and specialisation within the population and its effecting role on motor behaviour (e.g., Maraj, Robertson, Welsh, Weeks, Chua, Heath, Roy, Simon, Weinberg and Elliott, 2002; Elliott, Weeks and Elliott, 1987; Weeks, Chua and Elliott, 2000). A primary motivator for these studies has been the idea that atypical patterns of brain organisation found in persons with Down syndrome (Hart- ley, 1986; Pipe, 1988) could be responsible for some of the information processing difficulties experienced by persons from this population. For example, while persons with Down syndrome display many general cognitive problems, they also have difficulty performing tasks involving the perception, organisation and production of verbal material (Maraj et al., 2003).

Dichotic listening procedures were previously employed in initial neurobehavioural studies to examine cerebral specialisation for speech perception in Down syndrome (e.g., Bowler, Cufflin and Kiernan, 1985; Elliott, Weeks and Elliott, 1987; Elliott, Weeks and Chua, 1994). Dichotic listening procedures are a non-invasive means of examining cerebral specialisation for speech perception. In these studies, participants are typically presented with pairs of letters, digits or words simultaneously to the right and left ears through headphones. Participants can be asked to either recall all sounds heard or to report the sounds from one ear or the other. In these situations most right-handed children and adults will report more correct responses for the right ear than the left ear. The reason for this is that 
most auditory pathways are contra-lateral in set-up and as such the advantage of the right ear for the perception has been taken to be an indication that the left hemisphere is specialised for speech perception. The typical result when children with Down syndrome engage in dichotic listening procedures is that there is reversed hemispheric advantage for speech perception. That is, a left-ear right-hemisphere set-up (Chua, Weeks and Elliot, 1996).

Studies investigating cerebral development (e.g., Elliott, Edwards, Weeks, Lindley and Carnahan, 1987; Parlow, Kinsbourne and Spencer, 1996; Piccirilli, D’Alessandro, Mazzi, Sciarma, and Testa, 1991) have indicated that although persons with Down syndrome depend on their right hemisphere for speech perception, their left hemisphere appears to play the executive role in speech production (Maraj et al., 2002). Of relevance to the motor behaviour domain, left hemisphere specialisation for speech production is associated with a general lateralised proficiency for specifying the magnitude and timing of muscular force (Elliott and Chua, 1996). That is, persons with Down syndrome appear to perceive speech with their right cerebral hemisphere, but depend on their left cerebral hemisphere for the organisation and control of movement thus, exhibiting atypical patterns of brain organisation.

In the motor domain, relating to visual and verbal-motor development, persons with Down syndrome have demonstrated relative proficiency on skills involving the visual demonstration of movement (Edwards, Elliott and Lee, 1986; Frith and Frith, 1974; Le Clair and Elliott, 1995; Maraj et al., 2002). Several studies (Elliott, 1990; Elliott and Weeks, 1990; Elliott, Weeks and Gray, 1990; Welsh and Elliott, 2001) have shown that adults with Down syndrome exhibit more errors performing single manual oral gestures to a verbal command (e.g., "place your finger on your nose") than following the visual demonstration of a task. Elliott, Gray and Weeks (1991) proposed that the functional isolation of the speech perception (right hemisphere) and movement production (left hemisphere) systems has led to a breakdown in communication between these systems, adversely affecting tasks that require verbalmotor behaviour. This proposal had been previously formalised into a model of cerebral specialisation (Elliott, Weeks, Elliott, 1987).

Subsequent research based on this model has indicated that individuals with Down syndrome experience difficulties in performing motor tasks based on verbal instruction. The model has been used in accounting for the information processing difficulties on the basis of verbal instruction. Further, there is some evidence to suggest that persons with Down syndrome may consolidate visual information such that positive transfer is seen when they are switched from a visual to verbal mode of learning. Although much work has been done on simple upper limb movements, real progress toward influencing broader health and education practices demands that we assess gross motor skills. Gross motor skills are an important component of many physical activities. Moreover, the acquisition of these types of motor skills can facilitate many other activities of daily living. The purpose of this study was to examine gross motor skill acquisition in persons with Down syndrome based on visual and verbal instructional protocols.

\section{Method}

\section{Participants}

Participants were nine ambulatory persons with Down syndrome ( 6 males, 3 females). The participants were recruited from a daytime summer camp that provided a variety of sports and movement activities for persons with Down syndrome and was located at the Down Syndrome Research Foundation, British Columbia, Canada. Ages ranged from 13 to 23 years $(M=19, S D=3)$.

\section{Procedure}

Over a period of four consecutive days (Phase 1), participants were presented with three different gross motor skills (hop, step, jump). During this acquisition phase, an instructor taught the participants each of the three skills utilising both visual and verbal protocols. Phase 2 of the study was carried out on Day 5 (Baseline), where participants were individually video-recorded while performing each of the three skills. Each participant was requested to perform the skills separately and in isolation from other participants. If the participants did not perform the skill correctly on the first attempt, they were given feedback on their performance followed by the instructor explaining and demonstrating the skill to them. The participants then were given another attempt at executing the skill. The movement that was performed following this procedure was the movement analysed by the researcher. These video recordings served as baseline data for examination of skill transfer using either a visual or verbal instructional protocol during Phase 3 of the study.

Phase 3 of the study was carried out on Day 6, where the participants were randomly assigned to either a visual or verbal group (4 visual, 5 verbal). Participants in the visual group observed the instructor while they demonstrated the movement skills. Only one demonstration was provided per skill and participants were required to repeat the movement once only, and immediately following the instructors demonstration. The verbal group participants were prompted by the instructor to perform each of the individual skills. The instructor stated the skill (e.g., show me a hop) and the participant performed the movement. No other information was provided. No feedback was given to the participants upon the completion of the skills in either the visual or verbal group. One attempt was allowed for each skill performance. Video recording for Day 6 was carried out where participants did not view each other executing the skills. 


\section{Data analysis}

Prior to the video analysis of the skill execution, movement development sequences for the hop, step, and jump, were prepared by the researcher. The movement sequence for a hop has been partially validated by Halverson and Williams (1985). The movement sequence for a step has not been validated but adapted from Roberton and Halverson (1984). The movement sequence for a jump has not been validated and was adapted from Haywood (1993) and Kirchner and Fishburne (1995).

Each of the skills was broken into 3 separate components: arm, leg and trunk action. Each component contained ranked developmental steps. The first developmental step was the least mature (in terms of motor performance) and was ascribed a score of 1 , the next developmental step described a more mature level of skill and received a score of 2. Scoring continued in this fashion until the most mature step was reached which received the maximum score for the component. Scores for each component of a movement development sequence were added to ascribe the individual participants score for the skill. The maximum values for hop, step and jump were 12,12 and 25 , respectively. A zero score was assigned when a participant did not perform the skill in a particular fashion e.g., performed a hop instead of a jump, or the participant was unable to perform a component of a particular skill.

The video data collected for Phases 2 and 3 was then reviewed and each of the 3 movement skills was analysed and scored. This scoring was based on the developmental steps achieved within each component for each of the three skills as performed by the participants. Reliability of data analysis was confirmed by incorporating 3 inter-rater reliability reviews.

\section{Statistical analysis}

Statistical analysis was conducted using SPSS for Windows (release 10.0). Descriptive statistics were computed for all variables (hop, step and jump) on Day 5 and Day 6. An additional descriptive analysis of the data involved calculating the participants' skill scores on Days 5 and 6 of the study. This was determined as a percentage of the maximum (mature) value for that skill. Due to administering multiple comparisons a Bonferroni Adjustment was applied to the alpha level because multiple $t$ tests result in a greater probability of a Type I error. Alpha was calculated as $\mathrm{p} \leq 0.008$. Levene's test for equality of variances was applied to examine the homogeneity assumption. Subsequently, inferential statistics (independent samples $t$ test) were carried out.

\section{Results}

The data was analysed using both descriptive and statistical procedures. Levene's test of equality of variances indicated that no significant difference existed between groups on baseline measurement. Independent $t$ tests analysis indicated that no significant differences existed between groups at baseline (Day 5) for the three skills (see Figure 1). After receiving either visual or verbal instruction on day

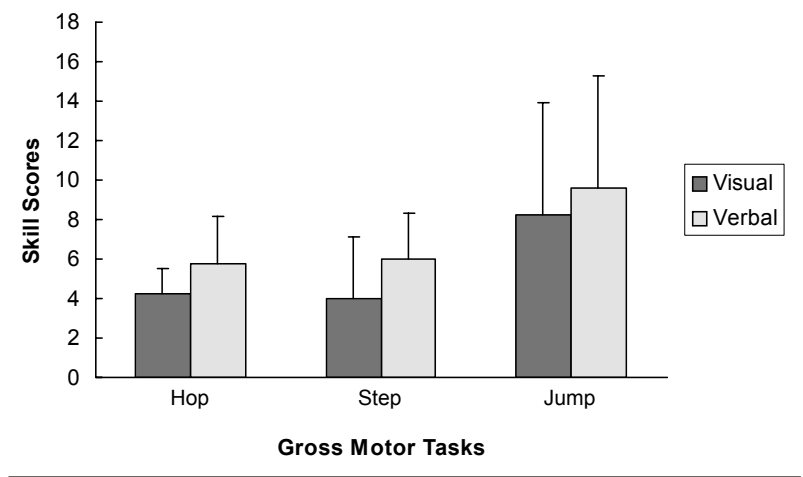

Figure I. Means and Standard Deviations of skill scores as a Function of Task and Group for Phase II (Baseline)

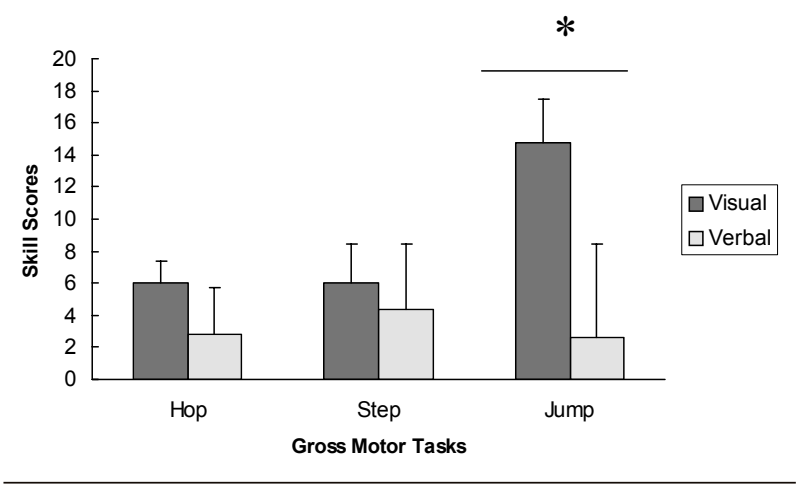

Figure 2. Means and Standard Deviations of skill scores as a Function of Task and Group for Phase III

6 , one significant difference was found between the groups for the jump skill performance $(t(7)=3.837, p<.006)$ at $\alpha$ level .008 (see Figure 2). The significant finding at the .008 level was seen as further support that the visual motor performance group displayed greater jump skill proficiency $(\mathrm{M}=14.75, \mathrm{SD}=2.63)$ than the verbal motor performance group $(M=2.6, S D=5.81)$ Statistical analysis found no significant difference between visual and verbal-motor performance for a hop and step on the instruction day.

The maximum mature skill scores for the hop, step and jump were 12, 12 and 25, respectively. Another finding in the current study was the observed differences from the baseline values to the instruction day values between the visual and verbal groups (based on the mean percentage of maximum mature skill scores). Results indicated a positive change as reflected by a higher mean percentage score in the performance of all three skills for the visual group when comparing Day 5 values to Day 6 values. In contrast, there was an overall decrease in the performance of all three skills for the participants in the verbal group from Day 5 to Day 6 (see Figure 3).

\section{Discussion}

The purpose of the present study was to investigate whether the verbal-motor performance deficits exhibited by individuals with Down syndrome (Elliott, 1990; Elliott and Weeks, 1990; Elliott, Weeks and Gray, 1990; Maraj et al., 


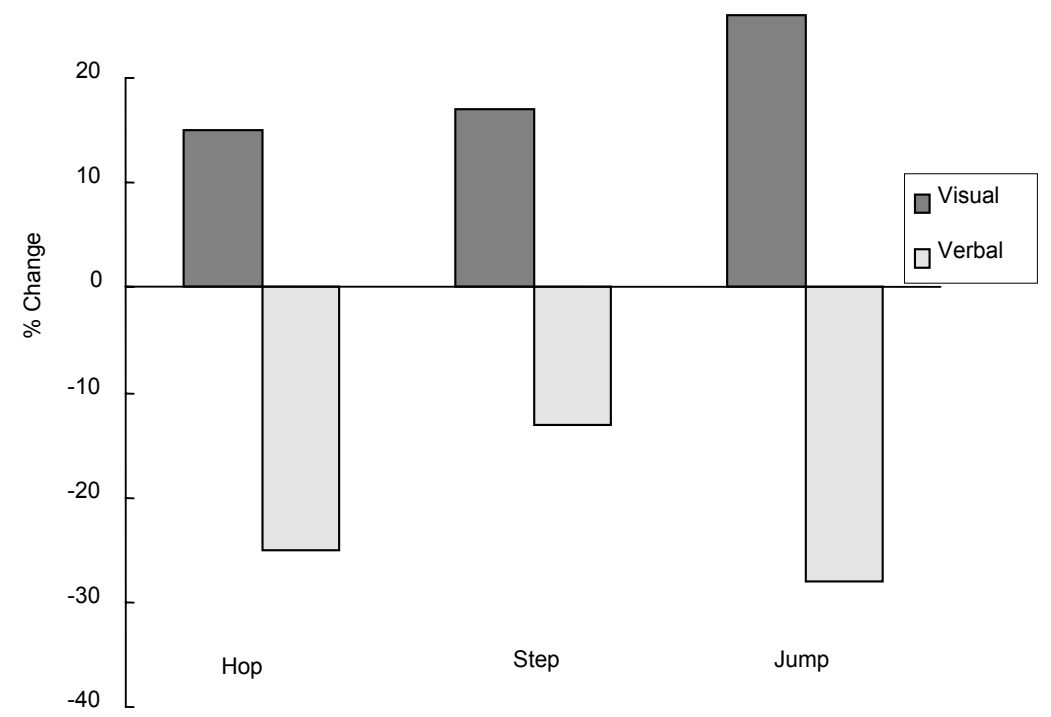

Figure 3. Percent change from Phase II to Phase III as a function of Group and Task

2002; Welsh and Elliott, 2001) limited their ability to transfer gross motor skills from visual and verbal instruction to either visual or verbal instruction. Participants were presented with three distinct motor skills and were required to reproduce the skills under visual or verbal instruction. The inferential results of the current study are partially consistent with the findings of previous studies based on visual and verbal-motor performance amongst the Down syndrome population and are particularly pertinent to Elliott et al. (1987) proposed model of atypical cerebral specialisation (see Chua, Weeks and Elliott (1996) for a review). The main tenet of this model is that areas responsible for speech perception are atypically specialised to the right hemisphere in persons with Down syndrome, while the left hemisphere is involved in the organisation and control of goal-directed movement. The model posits that persons with Down syndrome will have specific difficulty performing tasks that require both speech perception and movement organisation. This difficulty is predicted to result because these tasks are subserved by different cerebral hemispheres. Thus, because inter-hemispheric communication is required, persons with Down syndrome will exhibit longer information processing times and/or increased movement errors due to the degradation of information during neural transmission (Welsh and Elliott, 2001). Specifically, results of the present study provide partial support for the notion that individuals with Down syndrome have difficulty organising limb movement based on verbal instruction.

The results for the jump skill showed the most dramatic effect as the verbal group performed significantly poorer than the visual group. This type of behaviour strategy where "initiate the movement first, and then figure out the final destination while the movement is being completed" has been observed in individuals with Down syndrome (Welsh and Elliott, 2001, p.164). As a result of this, the verbal information to perform the skill (jump) may have still been processed and decoded throughout the skill exe- cution, thus resulting in more erroneous skill performance.

The unexpected finding of non-significance among verbal-motor performance in two of the presented motor skills stipulates that verbal-motor performance deficits were not exhibited and thus failed to limit the participants' skill transfer ability between visual and verbal instruction to verbal instruction only. This may be attributed to the concept termed 'transfer of learning'. This concept posits “the gain or loss of a person's proficiency on one task as a result of previous practice or experience on another task" (Schmidt and Wrisberg, 2000). Pertaining to the results found in the present study, one may argue that 'positive transfer' of the hop and step skills occurred as a consequence of 4 consecutive days (acquisition phase) of teaching and practice, followed by another consecutive day (Day 5) of skill performance under visual and verbal instruction. It is possible that the participants' prior experience in learning and practicing the skills had a beneficial effect on the transfer and performance of the hop and step when they were required to perform them under verbal instruction on Day 6. This is a concept known as generalisation or 'near transfer'. Near transfer is "a type of transfer of learning that occurs from one task to another very similar task or situation" (Schmidt and Wrisberg, 2000, p.179).

Contrastingly, participants may have displayed 'negative transfer' of the jump skill where prior experience was detrimental or non-influential when they were required to reproduce the skill the following day. It may be that the participants experienced cognitive difficulty under verbal instruction (as explained in the model of cerebral specialisation) in deciphering the difference between the skills e.g. a hop and jump, as individuals with Down syndrome exhibit general information processing difficulties as well as displaying a number of specific cognitive and motor problems when compared to other individuals with disabilities (Elliott, Gray and Weeks, 1991).

Visual and verbal-motor performances on Day 6 revealed a difference between visual and verbal-motor performance amongst the participants based on the mean percentage of maximum maturity skill scores. Specifically, results showed an increase in percentage mean scores for visual-motor performance for all 3 skills and a contrasting decrease in verbal-motor performance scores for the equivalent skills. This finding indicates that visual instruction facilitated in greater skill performance than verbal instruction. This concurs with the findings of previous research in the visual and verbal-motor domain, stipulating that individuals with Down syndrome tend to exhibit performance advantages under visual instruction when compared to verbal instruction (Edwards, Elliott and Lee, 1986; Frith and Frith, 
1974; Le Clair and Elliott, 1995; Maraj et al. 2002). Likewise, individuals with Down syndrome have demonstrated difficulty in performing tasks involving the perception, organisation and production of verbal material (Maraj et al., 2002). Once again, the finding of greater performance errors in verbal-motor behaviour may be attributed to Elliott et al. (1987) model of cerebral specialisation and Elliott, Gray and Weeks (1991) proposal that individuals with Down syndrome exhibit verbal-motor difficulties as a result of a dissociation of cerebral systems responsible for speech production and movement organisation.

In conclusion, the present study was an initial investigation into visual and verbal-motor behaviour amongst persons with Down syndrome utilising gross motor skills. It is fair to deduce that the verbal-motor performances deficits exhibited by individuals with Down syndrome (Elliott, 1990; Elliott and Weeks, 1990; Elliott, Weeks and Gray, 1990; Maraj et al., 2002; Welsh and Elliott, 2001) did indeed limit the participants' ability to transfer gross motor skills following visual and verbal instruction to verbal instruction only. The findings are in agreement with past studies relating to the visual and verbal-motor behaviour amongst the Down syndrome population. Moreover, the results ameliorate the proposal that individuals with Down syndrome perform relatively well on skills involving the visual demonstration of movement when compared to verbal-motor performance and longer-term retention of gross motor skills.

\section{Acknowledgements}

This work was supported by the Social Sciences and Humanities Research Council of Canada.

\section{Correspondence}

Dr. Brian Maraj • Perceptual Motor Behaviour Laboratory, Faculty of Physical Education and Recreation, University of Alberta, Edmonton, AB, Canada T6G 2H9

\section{References}

Bowler, D.M., Cufflin, J. \& Kiernan, C. (1985). Dichotic listening of verbal and non-verbal material by Down syndrome children and children of normal intelligence. Cortex, 21, 637-644

Chua, R., Weeks, D.J. \& Elliott, D. (1996). A functional systems approach to understanding verbal-motor integration in individuals with Down syndrome. Down syndrome, Research and Practice, 4, 25-36.

Edwards, J.M., Elliott, D. \& Lee, T.D. (1986). Contextual interference effects during skill acquisition and transfer in Down syndrome adolescents. Adapted Physical Activity Quarterly, 3, 250-258.

Elliott, D. (1990). Movement control and Down syndrome: A neuropsychological approach. In Elliott, D., Gray, S., \& Weeks, D. J. (1991). In Verbal cueing and motor skill acquisition for adults with Down syndrome. Adapted Physical Activity Quarterly, 8, 210-220.

Elliott, D. \& Chua, R. (1996). Manual asymmetries in goaldirected movement. In D. Elliott \& E.A. Roy (Eds).
Manual Asymmetries in Motor Performance (pp. 143 158). Boca Raton: CRC Press.

Elliott, D., Edwards, J.M., Lindley, S. \& Carnahan, H. (1987). Cerebral specialisation in young adults with Down syndrome. American Journal on Mental Retardation, 91, 480-485.

Elliott, D., Gray, S. \& Weeks, D. J. (1991). Verbal cueing and motor skill acquisition for adults with Down syndrome. Adapted Physical Activity Quarterly, 8, 210-220.

Elliott, D. \& Weeks, D.J. (1990). Cerebral specialization and the control of oral and limb movements for individuals with Down syndrome. Journal of Motor Behaviour, 22, 6-18.

Elliott, D., Weeks, D.J. \& Chua, R., (1994). Anomalous cerebral lateralization and Down syndrome. Brain and Cognition, 26, 191-195.

Elliott, D., Weeks, D.J. \& Elliott, C.L., (1987). Cerebral specialisation in individuals with Down syndrome. American Journal of Mental Retardation, 92, 3, 263-271.

Elliott, D., Weeks, D.J. \& Gray, S. (1990). Manual and oral praxis in adults with Down syndrome. Neuropsychologia, $12,1307-1315$

Frith, U. \& Frith, C.D. (1974). Specific motor disabilities in Down syndrome. Journal of Child Psychology and Psychiatry, 15, 293-301.

Halverson, L.E. \& Williams, K. (1985). Developmental sequences for hopping over distance: a prelongitudinal screening. Research Quarterly for Exercise and Sport, 56, 37-44.

Hartley, X.Y. (1986). A summary of recent research into the development of children with Down syndrome. Journal of Mental Deficiency Research, 30, 1-14.

Haywood, K.M. (1993). Life Span Motor Development (2nd ed.). Human Kinetics.

Johnson, J.T., Jr. \& Olley, J.G. (1971). Behavioural comparisons of mongoloid and non-mongoloid retarded persons: A review. American Journal of Mental Deficiency, 75, 546-559.

Kirchner, G. \& Fishburne, G. J. (1995). Physical Education for Elementary School Children ( $9^{\text {th }} \mathrm{ed}$.). Brown \& Benchmark.

Le Clair, D.A. \& Elliott, D. (1995). Movement preparation and the costs and benefits associated with advance information for adults with Down syndrome. Adapted Physical Activity Quarterly, 12, 239-249.

Maraj, B.K.V., Robertson, S.D., Welsh, T.N., Weeks, D.J., Chua, R.C., Heath, M., Roy, E.A., Simon, D.A., Weinberg, H.A. \& Elliott, D. (2002). Verbal-motor behaviour in persons with Down syndrome. In M. Cuskelly, A. Jobling \& S. Buckley (Eds) Down Syndrome Across the Lifespan. Pp. 175-193. London, England: Whurr Publishers Ltd.

Maraj, B.K.V., Li, L., Hillman, R., Jeansonne, J. \& Robertson, S.D. (2003). Verbal and visual instruction in motor skill acquisition for persons with and without Down syndrome. Adapted Physical Activity Quarterly, 20, 57-69.

Parlow, S.E., Kinsbourne, M. \& Spencer, J. (1996). Cerebral laterality in adults with severe mental retardation. Developmental Neuropsychology, 12, 299-312. 
Piccirilli, M., D’Alessandro, P., Mazzi, P., Sciarma, T. \& Testa, A. (1991). Cerebral organization for language in Down's syndrome patients. Cortex, 27, 41-47.

Pipe, M.E. (1988). Atypical laterality and retardation. Psychological Bulletin, 104, 343-349.

Roberton, M.A. \& Halverson, L.E. (1984). Developing children - their changing movement. Philadelphia: Lea \& Febiger. In Haywood, D.J. (1993). Life Span Motor Development ( $2^{\text {nd }} e d$.). Human Kinetics.

Schmidt, R. A. \& Wrisberg, C. A. (2000). Motor Learning and Performance. A Problem-Based Learning Approach $\left(2^{\text {nd }} e d\right.$.). Human Kinetics.

Weeks, D.J., Chua, R. \& Elliott, D. (Eds.). (2000). Perceptual Motor Behaviour in Down Syndrome. Champaign, IL: Human Kinetics.

Welsh, T.N. \& Elliott, D. (2000). The preparation and control of goal-directed limb movement in persons with Down syndrome. In Weeks, D. J., Chua, R. \& Elliott, D. (2000). Perceptual-Motor Behaviour in Down Syndrome. Human Kinetics.

Welsh, T.N. \& Elliott, D. (2001). The processing speed of visual and verbal movement information by adults with and without Down syndrome. Adapted Physical Activity Quarterly, 18, 156-167. 